\title{
New insight into two-color LIF thermometry applied to temperature measurements of droplets
}

\author{
A. Labergue $\cdot$ V. Deprédurand · A. Delconte • \\ G. Castanet $\cdot$ F. Lemoine
}

\begin{abstract}
When laser-induced fluorescence of droplets is used for measurements such as droplet temperature, a new dependence of the droplet size on the spectral distribution of fluorescence has been highlighted. The two-color laserinduced fluorescence technique applied to droplet temperature measurement requires a single fluorescent tracer and two spectral bands of detection for which the temperature sensitivity is different. Generally, the ratio of the intensities measured on each of the spectral bands of detection is assumed to be only temperature dependent. However, droplet dependence on diameter is also likely to influence the intensities ratio. This study provides some illustrations of the phenomenon, first on sprays with different mean statistical diameters and secondly on single droplets, for two temperature-sensitive fluorescent tracers in their solvents: sulforhodamine B dissolved in water and pyrromethene 597-8C9 dissolved in n-decane.
\end{abstract}

\section{Introduction}

The measurement of the temperature in a spray is an important issue in numerous engineering applications, e.g. droplet vaporization in internal combustion engines (Sazhin 2006; Maqua et al. 2008) or spray cooling of hot surfaces (Castanet et al. 2009). Few techniques can be used to characterize the local temperature of the liquid phase in a

A. Labergue $(\bowtie) \cdot$ V. Deprédurand · A. Delconte .

G. Castanet · F. Lemoine

LEMTA, Nancy-Université, CNRS,

2, Avenue de la forêt de Haye,

BP 160, 54504 Vandoeuvre-lès-Nancy, France

e-mail: alexandre.labergue@ensem.inpl-nancy.fr spray. The global rainbow thermometry (GRT) aims to smooth the sphericity effects usually found with the standard rainbow technique on single droplets (Vetrano et al. 2006; Van Beeck et al. 2003).

Other techniques are based on the laser-induced fluorescence of a tracer dissolved in the liquid to be sprayed as the use of an exciplex (Murray and Melton 1985; Gossage and Melton 1987) or the use of two fluorescent tracers (Sakakibara et al. 1997; Sakakibara and Adrian 1999 and Kim et al. 2003).

The two-color laser-induced fluorescence (LIF) technique was successfully applied to measure the mean temperature of single evaporating or combusting droplets (Lavieille et al. 2001; Castanet et al. 2003). The technique requires seeding the liquid with a temperature-sensitive fluorescent tracer. The ratio of the fluorescence signal measured on two spectral bands is assumed to depend only on temperature but not on the concentration of the added tracer, probe volume dimensions, laser intensity and optical layout. The extension of this technique, initially used on single droplets, to perform measurements in sprays, was not straightforward. The present paper aims to present further investigations into the influence of the droplet size on the spectral distribution of fluorescence on single droplets and in sprays. More, it is shown how the droplet size effect could be corrected to measure the temperature properly.

\section{State of art of two-color laser-induced fluorescence thermometry}

The main features of two-color laser-induced fluorescence thermometry are described in previous references (Lavieille et al. 2001; Lemoine et al. 1999). The technique 
consists in seeding the liquid to be sprayed with a low concentration of a temperature-sensitive fluorescent tracer, generally an organic dye. In the current paper, two tracers will be considered:

1. Sulforhodamine B (or kitton red), which is highly soluble in water.

2. Pyrromethene 597-8C9, which is not soluble in water but highly soluble in alkanes or in ketones and alcohols (Depredurand et al. 2008).

In the following, solution A will refer to the sulforhodamine $\mathrm{B}$ dissolved in water, and solution $\mathrm{B}$ to the pyrromethene 597-8C9 dissolved in n-decane. The fluorescence of these dyes can be induced by the green line of a CW $\operatorname{argon}$ ion laser $(\lambda=514.5 \mathrm{~nm})$. Both fluorescence spectra are broadband and have a temperature-dependent shape. The fluorescence intensity, collected on a spectral band $i$ was expressed by (Lavieille et al. 2001):

$I_{f_{i}}=K_{\mathrm{opt}, i} K_{\mathrm{spec}, i} V_{c} I_{o} C f_{i}(T)$

where $K_{\mathrm{opt}, i}$ is an optical constant, $K_{\mathrm{spec}, i}$ is a constant that characterizes the fluorescence properties of the tracer in its solvent, $I_{o}$ the laser excitation intensity, $C$ the tracer concentration, $T$ the absolute temperature, $V_{c}$ the fluorescence probe volume. This latter is defined as the intersection between the illuminated part of the droplet volume and the field of view for the detection optics. The function $f_{i}(T)$ describes the temperature dependence of the emitted fluorescence on the spectral band $i$ and can be approximated as (Castanet et al. 2003):

$f_{i}(T) \approx e^{\frac{a_{i}}{T}+\frac{b_{i}}{T^{2}}}$

$a_{i}$ and $b_{i}$ are two coefficients that are determined experimentally. To properly measure the temperature of a moving droplet, the influence of the unknown and variable parameters $\left(C, V_{c}\right.$ and $\left.I_{o}\right)$ must be removed. In order to remove these problems, the fluorescence signal is detected simultaneously on two spectral bands for which the temperature sensitivity is sufficiently different. According to Eq. (1), it is possible to derive a ratio between the intensities collected on each spectral band, which depends only on temperature:

$R_{12}=\frac{I_{f 1}}{I_{f 2}}=\frac{K_{\mathrm{opt}, 1} K_{\mathrm{spec}, 1} f_{1}(T)}{K_{\mathrm{opt}, 2} K_{\mathrm{spec}, 2} f_{2}(T)}=\frac{K_{\mathrm{opt}, 1} K_{\mathrm{spec}, 1}}{K_{\mathrm{opt}, 2} K_{\mathrm{spec}, 2}} e^{\frac{a_{1}-a_{2}}{T}+\frac{b_{1}-b_{2}}{T^{2}}}$

Generally, the temperature measurement is based on the comparison between the actual fluorescence ratio and a fluorescence ratio $R_{120}$ that is measured under controlled conditions at a known temperature $T_{0}$. In this way, both constant $K_{\mathrm{opt}, i}$ and $K_{\mathrm{spec}, i}$ can be removed. The absorption and emission spectra for both solutions A and B are given in Fig. 1.
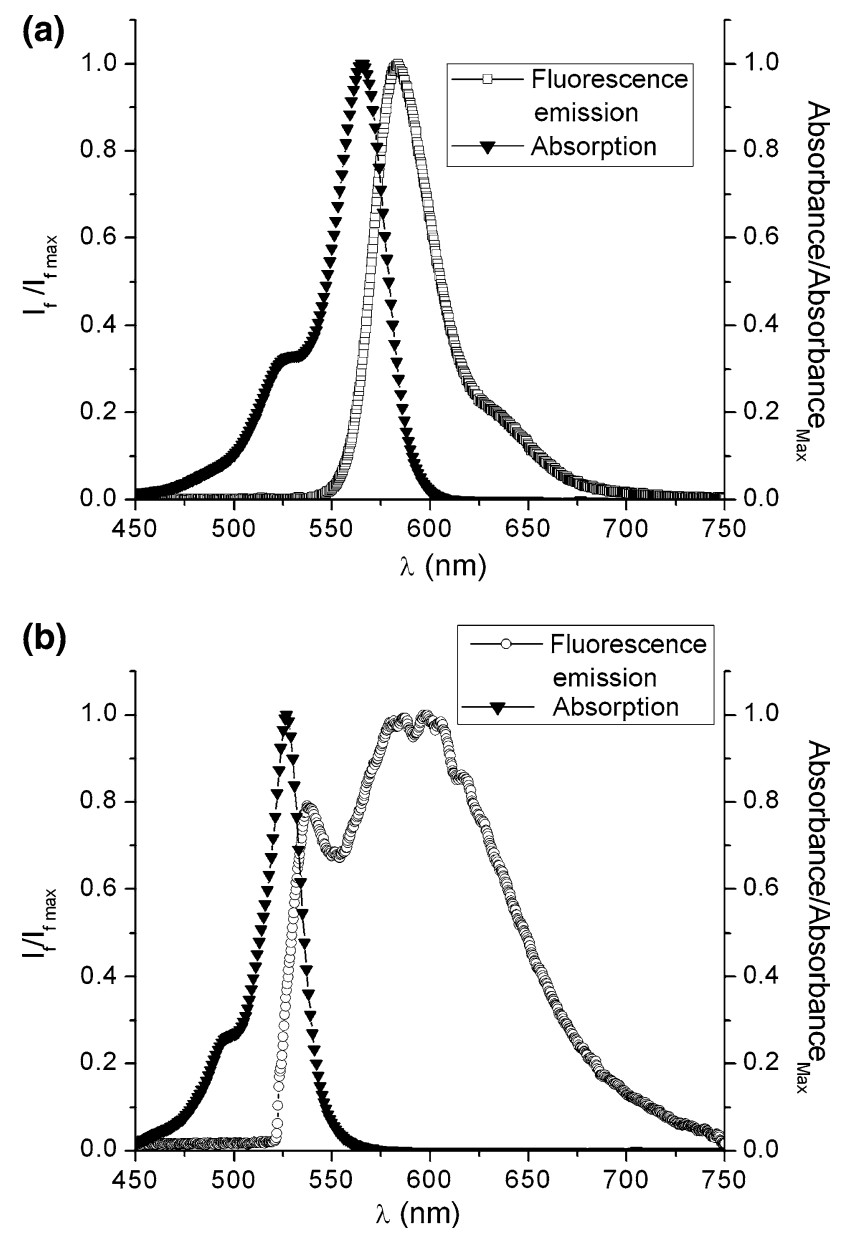

Fig. 1 Absorption and fluorescence emission spectra for both fluorescent tracers: sulforhodamine B in water (a) and pyrromethene 597-8C9 in n-decane (b)

The selection of these spectral bands was optimized in order to increase the temperature sensitivity of the intensity ratio $R_{12}$. The selected spectral bands are summarized in Table 1.

A calibration temperature is required in order to determine the temperature response of both solutions A and B, i.e. to determine the value of both coefficients $\left(a_{1}-a_{2}\right)$ and $\left(b_{1}-b_{2}\right)$ in Eq. (3). This calibration is undertaken in a controlled temperature cell, made in borosilicate glass, with the same set-up as Depredurand et al. (2008). This cell is also used for the measurement of the reference $R_{120}$ where the temperature $T_{0}$ is obtained with the help of a $K$ type thermocouple. The temperature sensitivity is about

Table 1 Spectral bands used for both solutions A and B

\begin{tabular}{lll}
\hline & Solution A & Solution B \\
\hline Band 1 & {$[555 \mathrm{~nm} ; 575 \mathrm{~nm}]$} & {$[540 \mathrm{~nm} ; 560 \mathrm{~nm}]$} \\
Band 2 & $\lambda>615 \mathrm{~nm}$ & {$[590 \mathrm{~nm} ; 610 \mathrm{~nm}]$} \\
\hline
\end{tabular}


Fig. 2 Spray facilities and optical devices for the fluorescence signal detection

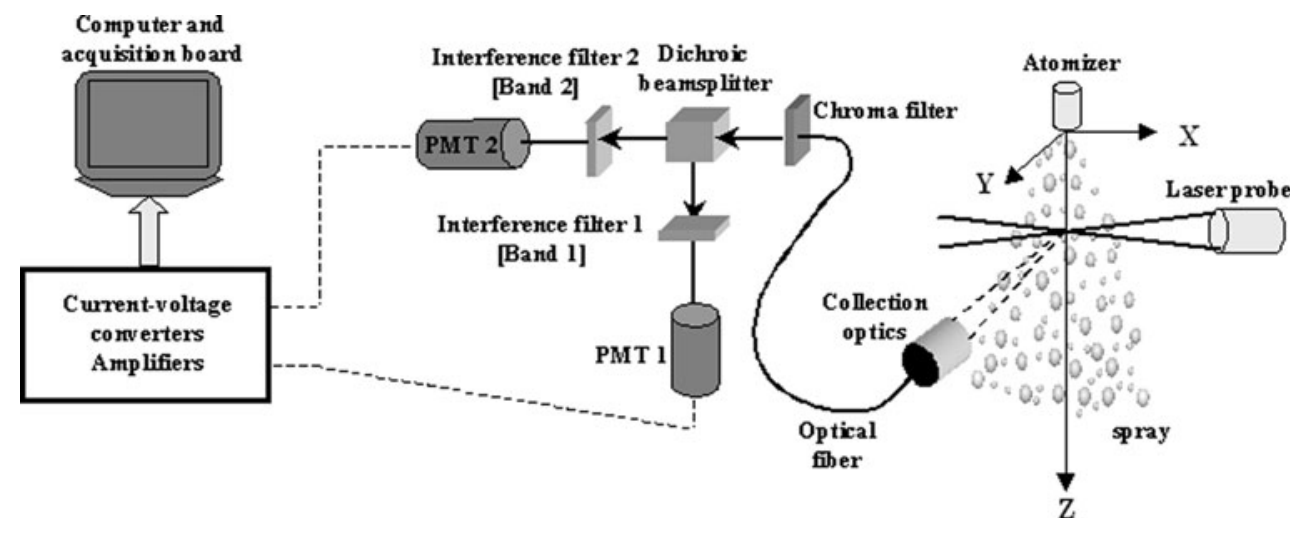

$0.9 \% / \mathrm{K}$ for solution $\mathrm{A}$ and $0.8 \% / \mathrm{K}$ in the case of solution B.

\section{Use of two-color LIF thermometry in sprays}

\subsection{Experimental set-up}

\subsubsection{Spray facility}

The spray is obtained with the help of a Danfoss ${ }^{\circledR}$ atomizer supplied by a pressurized liquid tank. The pressure varies from 1 to about 6.5 bar $( \pm 0.1$ bar). The spray is injected vertically downward, along the $Z$ axis, at atmospheric pressure and room temperature (Fig. 2). The origin of the vertical axis $(Z)$ is taken at the atomizer exit.

\subsubsection{Optical set-up and date processing}

The laser excitation volume required for droplet size and LIF measurements is generated by a Laser Dopller Anemometry probe (Dantec ${ }^{\circledR}$ Fiber-Flow probe) with a focal length of $310 \mathrm{~mm}$. The laser source is an argon ion laser tuned on the green line at $\lambda=514.5 \mathrm{~nm}$. Thus, the laser excitation volume dimension is $2003 \mu \mathrm{m}$ long in $X$ direction and $146 \mu \mathrm{m}$ in diameter ( $Z$ direction).

The fluorescence signal is collected at right angle by an achromatic doublet coupled with an optical fiber (core diameter, $70 \mu \mathrm{m}$ ). Taking into consideration the magnification of this device and its depth of field, the fluorescence probe volume $V_{m}$, being the intersection of the collection and excitation volumes, is $210 \mu \mathrm{m}$ long in the $X$ direction and $146 \mu \mathrm{m}$ large in the $Z$ direction, i.e. $V_{m} \approx 0.003 \mathrm{~mm}^{3}$.

Droplet size distribution is obtained with the use of a classic PDA probe (Phase Doppler Anemometry; Dantec ${ }^{\circledR}$ Dynamics) operating in the refraction mode. A typical droplet size histogram of the spray, recorded at $Z=13 \mathrm{~mm}$ for an injection pressure $P=4$ bars, is represented in Fig. 3 for both solutions A and B. It appears that solution B leads to smaller droplets than solution A. Indeed, the mean

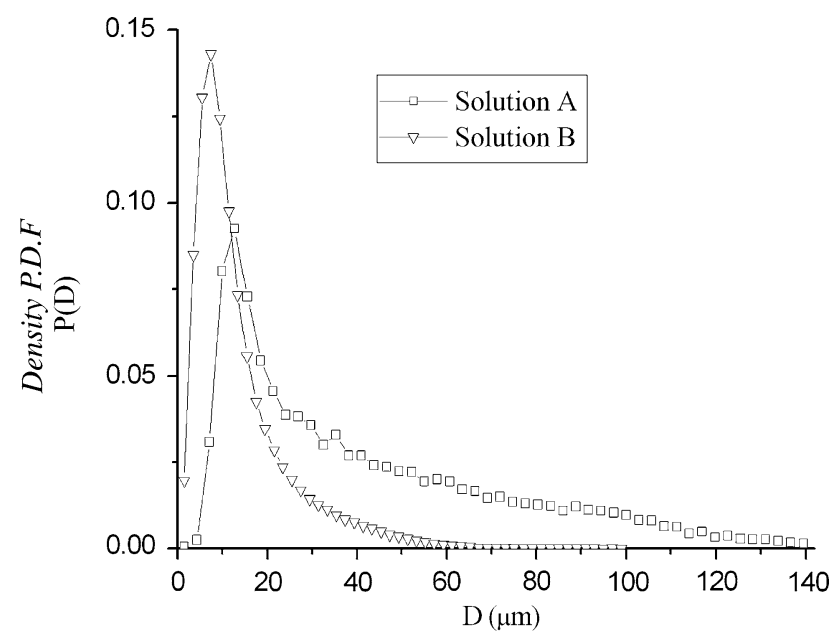

Fig. 3 Typical size histogram for both solutions obtained with a Danfoss ${ }^{\circledR}$ atomizer for an injection pressure of 4 bar

diameter for solution $\mathrm{A}$ and $\mathrm{B}$ is respectively 70 and $50 \mu \mathrm{m}$. Next, the optical signal is high pass filtered by a Chroma ${ }^{\circledR}$ filter (HQ522LP) in order to remove the light at the laser wavelength. The remaining fluorescence signal is split into the two spectral bands of interest by means of a set of dichroic and interference filters (Fig. 2). The fluorescence signal is detected by means of two photomultiplier tubes and digitalized by a multi-channel acquisition board. The signal issuing from the two photomultipliers is digitalized with a frequency of $5 \mathrm{MHz}$ and stored in a buffer for real time processing.

Prior to the measurements, the noise level of the detection chain (PMTs and amplifier) is determined by carrying out a measurement without laser light. Then, a threshold is fixed significantly above the noise level of the respective channels of detection. If 10 consecutive samples have values above the threshold, these sequences of samples are considered to become droplets. The fluorescence ratio is not determined for an individual droplet, due to the signal to noise ratio, caused by the random arrival of the photons on the photocathode, following a Poisson statistics (Gaskey et al. 1990). An averaging process is implemented, 
and the ratio of the fluorescence intensity on the two spectral bands 1 and 2 is calculated as follows:

$R_{12}=\frac{\sum_{j=1}^{N} I_{f j 1}}{\sum_{j=1}^{N} I_{f j 2}}$

where $I_{f j 1}$ and $I_{f j 2}$ are respectively the fluorescence intensities integrated on the $j$ th droplet crossing the probe volume, for the spectral bands 1 and 2. $N$ is the number of droplets detected during the acquisition. In a designated spray area defined by the probe volume, droplets that may have different temperatures are involved in the temporal averaging process. Then, a local mean temperature could be determined. Here, we assume that only one droplet can be present in the probe volume $V_{m}$ for each position in the spray. Indeed, PDA measurements can lead to know the droplet concentration $C_{d}$. Of course, $C_{d}$ decreases with the distance from the atomizer exit and with the injection pressure. During our experiments, $Z=13 \mathrm{~mm}$ is nearest point from the atomizer and $P=7$ bars is the greatest pressure used. In such case, $C_{d}=55$ droplets per $\mathrm{mm}^{3}$. Thus, there is less of one droplet in $V_{m}$. We can conclude there is never more one of droplet within the probe volume.

\subsection{Experiments in the spray}

\subsubsection{Preliminary experiments}

In a first step, the fluorescence ratio was measured in the spray, at $Z=15 \mathrm{~mm}$, at different injection pressures for both solutions A and B. For the considered spray, the droplets are often smaller than the probe volume area. Then, saturation effects that could result from droplets exceeding the probe volume area are not expected. For solution A, dye concentration is $C=5 \times 10^{-6} \mathrm{~mol} / \mathrm{l}$ and is $C=10^{-6} \mathrm{~mol} / \mathrm{l}$ for solution B. At this position (rather close the nozzle), the temperature variations are very limited, and the temperature can be considered as fairly independent on the injection pressure and almost equal to the injection temperature. These results, following the data processing of Eq. (4), are then compared to the fluorescence ratio $R_{120}$ measured in a cell with the same optical conditions (i.e. PMT high voltages, optical components) and at the same temperature. Figure 4 describes the evolution of the normalized ratio $R_{12} / R_{120}$ versus the injection pressure. The normalized fluorescence ratio varies significantly with the injection pressure and is very different from the ratio measured in the cell (i.e. $R_{12}=R_{120}$ ). This behavior is not predicted by the model of Eq. (1) and must be thoroughly investigated.

As a significant overlap between absorption and emission spectra of the fluorescence tracer exists, the fluorescence signal could be re-absorbed along the optical path

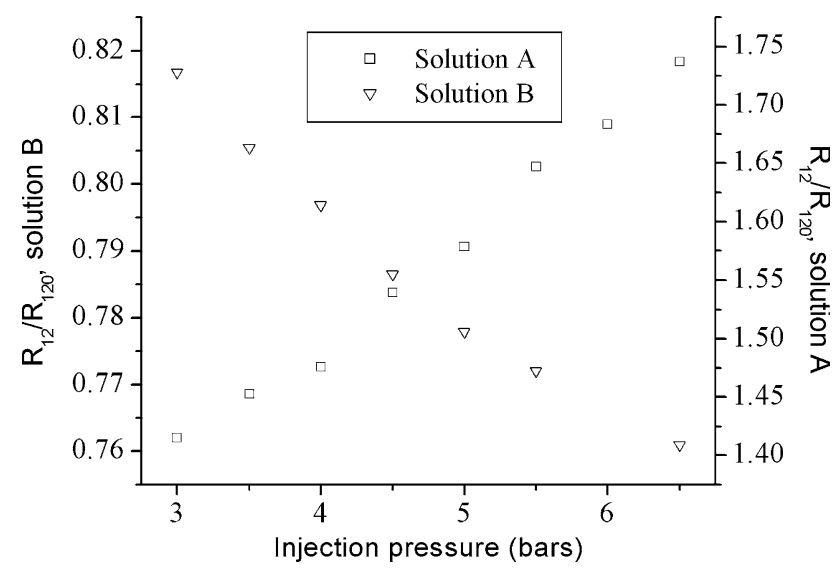

Fig. 4 Evolution of the normalized fluorescence ratio as a function of the injection pressure for solution A and solution B

between the laser excitation volume and the collection optics. Therefore, using the droplet number concentration $C_{d}$ given by PDA with the optical path and tracer concentration, it is possible to estimate an effective optical path which corresponds to an optical path where only liquid occurs between the probe volume and the collection optics. Its value is then about $13.8 \mu \mathrm{m}$. Knowing the molar extinction coefficients corresponding to the two spectral bands of detection $\left(\varepsilon_{1}=2.2 \times 10^{7} \mathrm{~mol}^{-1} 1 \mathrm{~m}^{-1}\right.$ and $\varepsilon_{2}=3 \times 10^{5} \mathrm{~mol}^{-1} 1 \mathrm{~m}^{-1}$ ), the Beer's Lambert law leads to a modification of the fluorescence ratio of about $0.2 \%$.

\subsubsection{Effect of the droplet size distribution}

The effect of the droplet size distribution on the fluorescence ratio was also investigated, since the injection pressure of the liquid modifies the droplets size distribution. In the case of solution A, several atomizers, including the Danfoss ${ }^{\circledR}$ atomizer used previously, under different injection pressures, have been operated to obtain different droplet size distributions. In the following, the statistical diameter $D_{30}$, measured with the use of the PDA, was used to characterize the droplet size distribution. The statistical diameter $D_{30}$ corresponds to a droplet that has the mean volume of the droplets in the distribution (Lefebvre 1989). This diameter has been preferred in order to taking into account that the fluorescence signal evolves proportionally with the droplet volume (Eq. 1). Figure 5 depicts the variation of the normalized fluorescence ratio $R_{12} / R_{120}$ with the statistical diameter $D_{30}$. The normalized ratio decreases as $D_{30}$ increases and tends to the ratio measured in a cell $\left(R_{12}\right)$ $\left.R_{120}=1\right)$ for the biggest statistical diameters. In parallel, fluorescence spectra have been recorded, with a resolution of about $1 \mathrm{~nm}$, for different statistical diameters $D_{30}$ and compared to a spectrum recorded in a cell (Fig. 6). The spectrum was recorded in the cell with a special care in order 


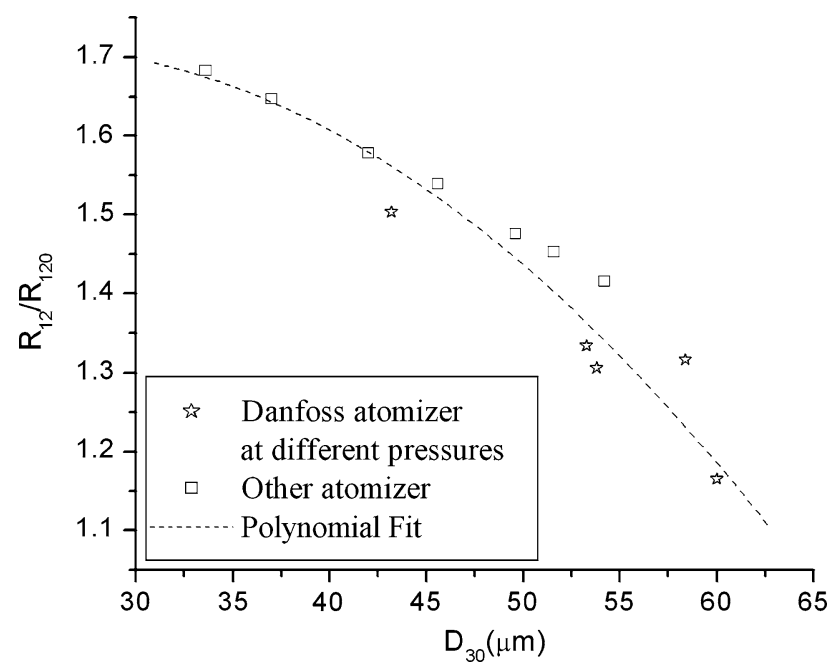

Fig. 5 Evolution of the fluorescence ratio as a function of the statistical diameter $D_{30}$ for solution A, obtained with different atomizers

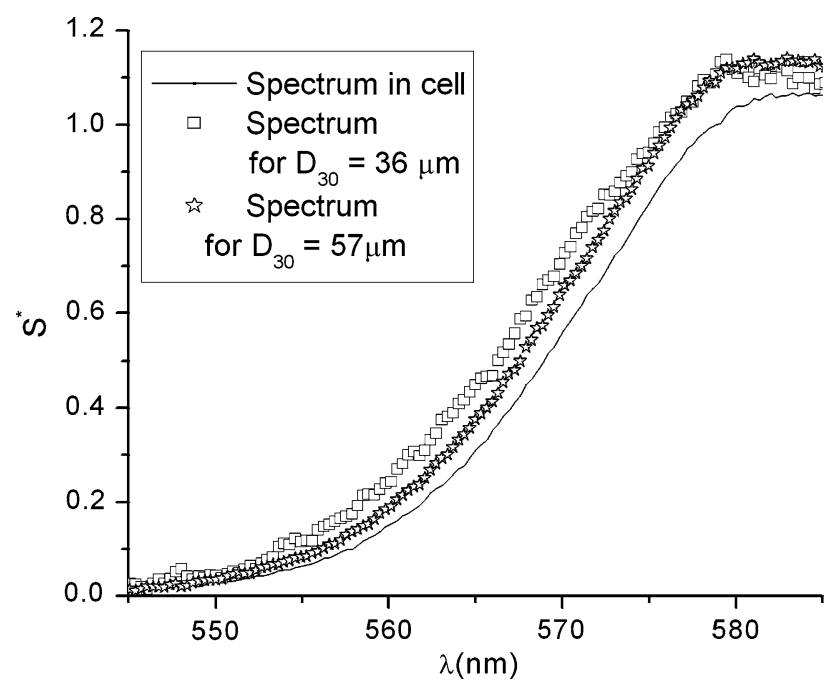

Fig. 6 Fluorescence spectra of solution A, recorded in the spray for two mean statistical diameters $D_{30}$ and comparison with the spectra recorded in a cell

to minimize the optical path between the laser beam and the glass wall of the cell. For instance, the influence of the reabsorption of the fluorescence emission is about $1 \%$ on the fluorescence ratio for an optical path of $97 \mu \mathrm{m}$, when the tracer concentration is $C=5 \times 10^{-6} \mathrm{~mol} / \mathrm{l}$ (solution A). To make the comparison, spectra were normalized by their own intensity integrated on a spectral band, where the effect of the droplet size distribution is not significant, as followed:

$S^{*}(\lambda)=\frac{S(\lambda)}{\int_{\lambda_{1}}^{\lambda_{2}} S(\lambda) d \lambda}$

where $\lambda_{1}=685 \mathrm{~nm}$ and $\lambda_{2}=700 \mathrm{~nm}$ in the present case (solution A). Figure 6 is reduced to the region of the spectra where the most significant changes can be
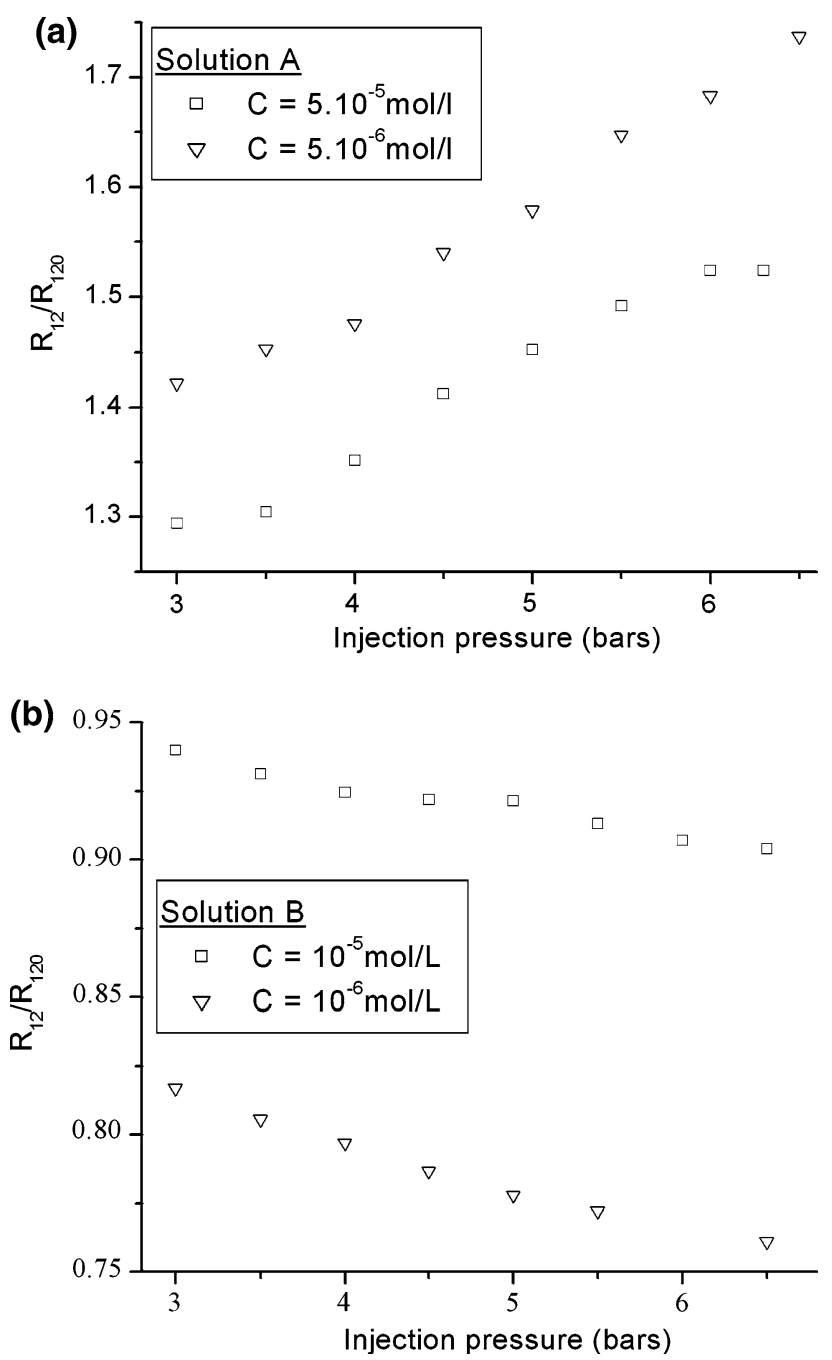

Fig. 7 Effect of the tracer concentration on the evolution of the fluorescence ratio with the injection pressure for solution $\mathrm{A}$ (a) and solution B (b)

observed. The spectrum corresponding to the smallest statistical diameter $\left(D_{30}=36 \mu \mathrm{m}\right)$ appears significantly blue-shifted while the spectrum for $D_{30}=57 \mu \mathrm{m}$ is closer to the one measured in the cell.

\subsubsection{Effect of the dye concentration}

The effect of the dye concentration was also investigated. It was increased by a factor 10 for both solutions A and B (new dye concentrations being respectively $C=5 \times 10^{-5} \mathrm{~mol} / \mathrm{l}$ for the solution A and $C=10^{-5} \mathrm{~mol} / \mathrm{l}$ for the solution B). The fluorescence ratio was measured in the case of the Danfoss ${ }^{\circledR}$ atomizer for different injection pressures. These measurements were superimposed with the ones obtained at the lower concentration for solution A (Fig. 7a) and for solution B (Fig. 7b). It appears clearly that the variation of the fluorescence ratio with the injection pressure is 
Table 2 Decrease of the fluorescence variation in \% due to the dye concentration for both solutions A and B

\begin{tabular}{llll}
\hline & Solution A & & Solution B \\
\hline$C=5 \times 10^{-5} \mathrm{~mol} / 1$ & $16.8 \%$ & $C=10^{-5} \mathrm{~mol} / 1$ & $2.9 \%$ \\
$C=5 \times 10^{-6} \mathrm{~mol} / 1$ & $19.5 \%$ & $C=10^{-6} \mathrm{~mol} / 1$ & $5.5 \%$ \\
\hline
\end{tabular}

significantly reduced for the highest dye concentration over the investigated pressure range [3.0; 6.5 bars] (see Table 2). The fluorescence ratio is also closer to the one measured in a cell.

However, when dye concentration is significantly increased, the self-quenching phenomenon can influence the fluorescence quantum yield according to Perrin's law (Bruhat 1992; Bazile and Stepowski 1994). In order to limit the influence of self-quenching to $1 \%$ in the case of solution $\mathrm{A}$, the dye concentration $C$ should not exceed $6 \times 10^{-3} \mathrm{~mol} / \mathrm{l}$, which is far from the present dye concentrations. It could be also anticipated that the behavior will be coarsely similar for solution B.

\section{Experiments on calibrated single droplets}

In order to investigate more precisely the respective influence of the droplet size and dye concentration effects, further experiments have been carried out on calibrated single droplets.

\subsection{Experimental set-up}

A monodisperse droplet generator was used to carry out specific experiments on calibrated droplets. It is based on the disintegration of a liquid jet undergoing a Rayleightype instability due to vibrations generated by a piezoelectric ceramic. For specific frequencies of the piezoceramic, the liquid jet breaks up into equally spaced and monosized droplets (Koenig et al. 1986). By adjusting the liquid flow rate, the piezoceramic frequency and the size of the outlet orifice, it is possible to obtain a wide range of droplet diameters ranging from 40 to $350 \mu \mathrm{m}$. If necessary, the fuel can be pre-heated in the injector body by means of a heat exchanger supplied by circulating temperature regulated water. The temperature of the fuel can be measured at the injection point by means of a $K$ type thermocouple. The measurement setup and data-processing of the monodisperse droplets is similar to the one implemented in sprays. The measurements are generally based on the averaging of 10,000 droplets.

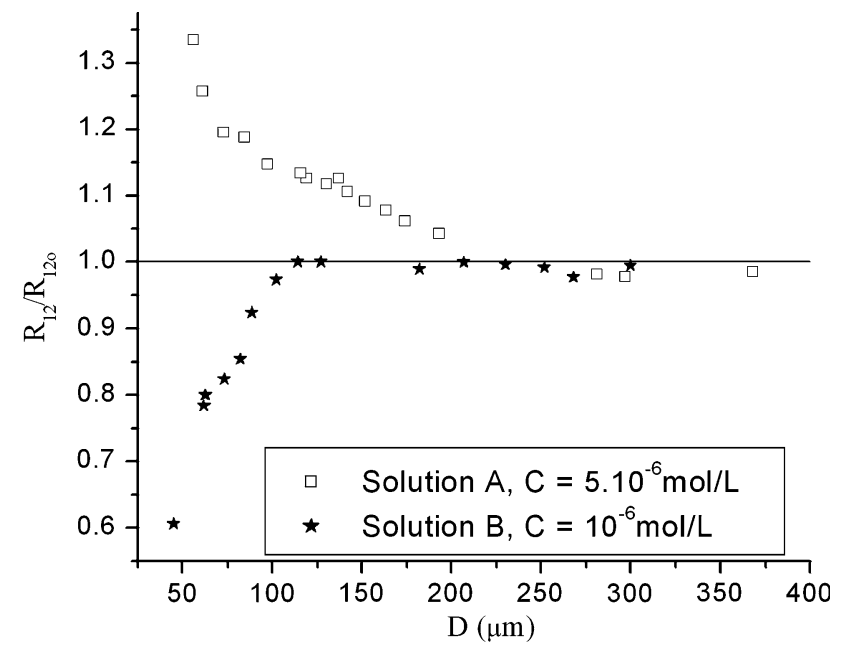

Fig. 8 Evolution of the fluorescence ratio as a function of the droplet diameter of calibrated single droplets for solutions A and B

\subsection{Influence of the droplet size}

The influence of the droplet size was tested for both solutions $\mathrm{A}$ and $\mathrm{B}$, with respective dye concentrations $C=5 \times 10^{-6} \mathrm{~mol} / \mathrm{l}$ and $C=10^{-6} \mathrm{~mol} / \mathrm{l}$. The measurements are performed $10 \mathrm{~mm}$ after the injector exit, after the liquid jet breaks into droplets and when spherical droplets are obtained. The low volatility of both liquids allows avoiding significant temperature variations caused by evaporation.

The fluorescence ratio was measured for droplet diameters $D$ ranging from 45 to $360 \mu \mathrm{m}$ for both solutions A and $\mathrm{B}$ and normalized by a reference that corresponds to the ratio measured in a cell. The experimental results are reported in Fig. 8 for both tested dyes. For solution A, the fluorescence ratio is almost constant for droplet diameters higher than $D=200 \mu \mathrm{m}$ and rises progressively when the diameter decreases. For the biggest droplets, the fluorescence ratio appears under the reference measurement. This phenomenon can be attributed to the signal trapping due to re-absorption (Fig. 1) that subsequently modifies the fluorescence ratio. Indeed, using Beer's Lambert law and taking a droplet diameter $D$ of $250 \mu \mathrm{m}$ as an optical path and a dye concentration $C$ of $5 \times 10^{-6} \mathrm{~mol} / \mathrm{l}$, the re-absorption of the fluorescence ratio could be expressed as:

$\frac{R_{12}}{R_{120}}=e^{-\left(\varepsilon_{1}+\varepsilon_{2}\right) C D}$

and its value is about $0.975 \%$. Here, both molar extinction coefficients $\varepsilon_{1}$ and $\varepsilon_{2}$ are the same as in previous Sect. 3.2.1. Thus, the decrease of the fluorescence ratio $R_{12}$ compared to those in cell $R_{120}$ is about $2.5 \%$. This value is well in consistent with results observed in Fig. 8 for $D>200 \mu \mathrm{m}$. For solution B, the fluorescence ratio is constant and equal to the reference measurement for 
diameters almost higher than $100 \mu \mathrm{m}$ and then decreases slightly for smaller droplet diameters. Fluorescence spectra, normalized following Eq. (5), are recorded for two diameters with droplets made of solution A (Fig. 9a) and solution B (Fig. 9b). In the latter, $\lambda_{1}=517 \mathrm{~nm}$ and $\lambda_{2}=534 \mathrm{~nm}$ were chosen for the normalization, always where the size effect is negligible, although re-absorption appears in this wavelength range (Fig. 1b). Nevertheless, taking the biggest droplet diameter (i.e. $D=202 \mu \mathrm{m}$ here) and $C=10^{-6} \mathrm{~mol} / \mathrm{l}$, Eq. (6) in this wavelength range leads to a fluorescence ratio decrease of about $2 \%$. Thus, the way to normalize the spectra is very weakly affected by reabsorption phenomena. The spectrum measured in a cell at the same temperature is also superimposed.

In the case of solution A, a blue shift is observed as in the spray for the smallest droplet size droplets and the spectrum in the cell coincides roughly with the one of the
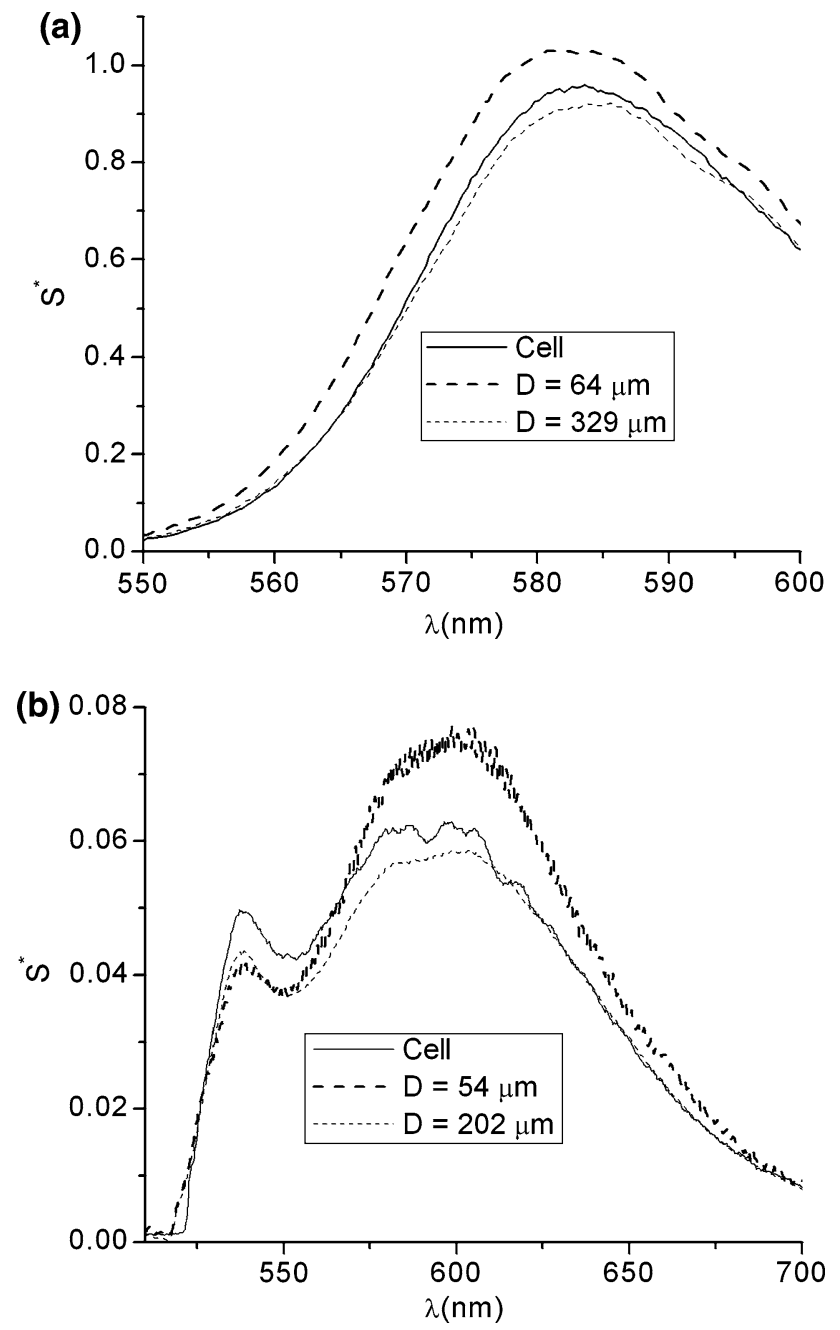

Fig. 9 Fluorescence spectra recorded with calibrated single droplets for two droplet diameters (solution A (a) and solution B (b)). Comparison with the spectrum obtained in a cell biggest droplet. Concerning the solution $\mathrm{B}$, spectra exhibit two peaks. We can observe that the droplet size effect is more important for the second peak (i.e. at about $600 \mathrm{~nm}$ ). Furthermore, as stated for solution A, the spectrum corresponding to the highest droplet diameter is closer to the spectrum measured in the cell.

\subsection{Influence of the dye concentration}

As it was done in the spray, the dye concentration in the droplets was increased by a factor 10 (Fig. 10). Compared to the evolution of the ratio at the lower dye concentration (Fig. 8), the influence of the droplet size on the fluorescence ratio appears significantly reduced for small droplets diameter, especially for $D<100 \mu \mathrm{m}$. In the case of solution $\mathrm{A}$, the values of the fluorescence ratio are very close to the reference measurement $\left(R_{12} / R_{120}=1\right)$ for diameters less than $100 \mu \mathrm{m}$. Over $D=100 \mu \mathrm{m}$, the ratio is clearly under the reference, which can be attributed to strong reabsorption phenomena, this phenomenon being particularly important on the first spectral band (Fig. 1a) for this high dye concentration. For solution B, normalized fluorescence ratio are almost about $4 \%$ lower than the reference measurement for diameters less than $100 \mu \mathrm{m}$ and equal to 1 for greater diameters. As the overlap between emission and absorption spectra is narrow (Fig. 1b), the re-absorption of the fluorescence is very low. That's why no re-absorption is observed (i.e. no points with $R_{12} / R_{120}<1$ ).

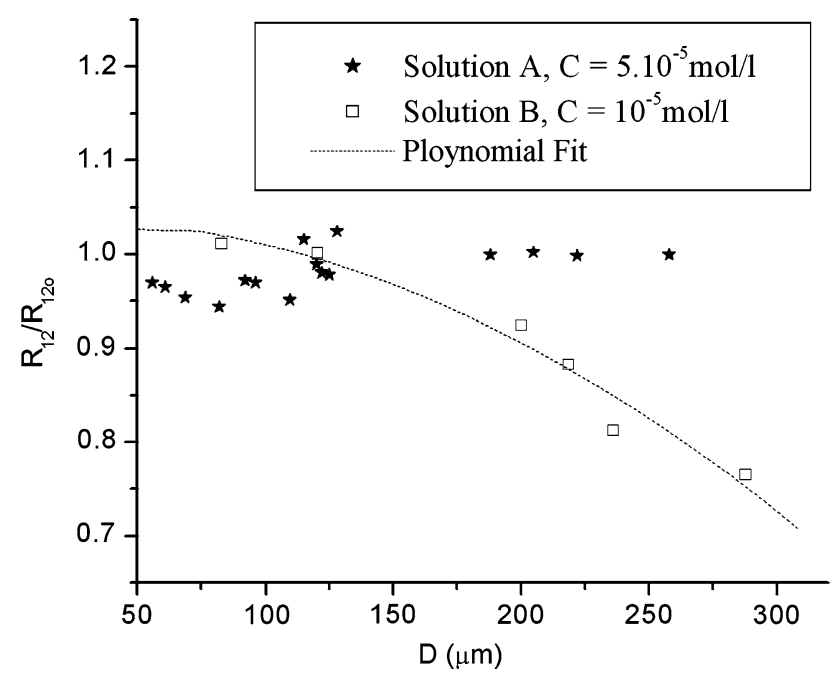

Fig. 10 Evolution of the fluorescence ratio as a function of the diameter of single droplets for both solutions A and B with increased dye concentrations 


\section{A new insight into the fluorescence emission of a droplet}

From the previous observations, the model of Eq. (1) must be corrected. Experiments reveal that the effects of the dye concentration and the droplet size cannot be separated. As an attempt, it has been assumed that the crossed influence of dye concentration and droplet size can be described by a single parameter $C^{\alpha} D^{\beta}$. A simple dimensional analysis leads to $\alpha=1 / 3$ and $\beta=1$. In practical words, it means that increasing the dye concentration by a factor 10 would be equivalent to increase the droplet diameter by a factor 2.15. To check the reliability of this assumption, $R_{12} / R_{120}$ was plotted against the parameter $C^{1 / 3} D$ for all the investigated range of droplet diameters and the two dye concentrations, for solutions $\mathrm{A}$ and $\mathrm{B}$ (Fig. 11a and $\mathrm{b}$ respectively) at ambient temperature $T_{0}=26^{\circ} \mathrm{C}$. For each of the solutions $\mathrm{A}$ or $\mathrm{B}$, it appears that the parameter $C^{1 / 3} D$ allows
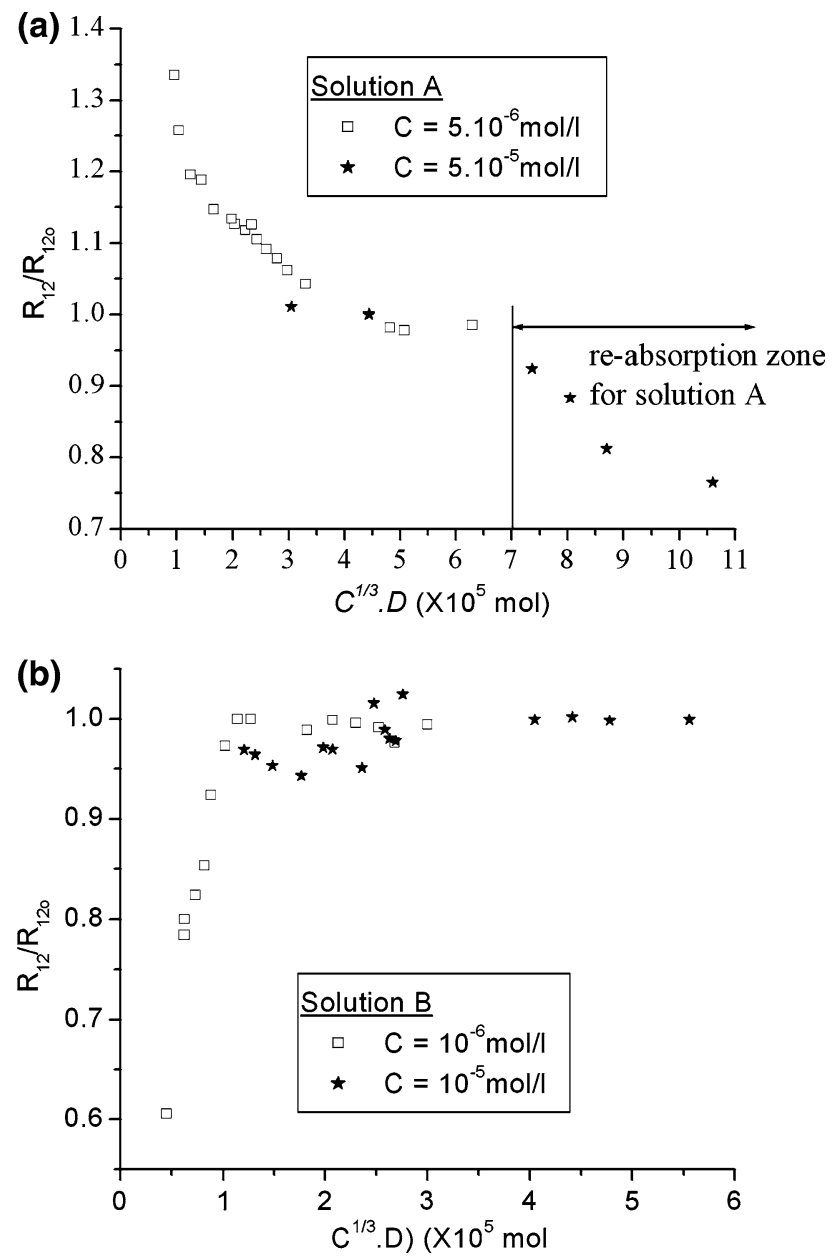

Fig. 11 Evolution of the fluorescence ratio as a function of the parameter $C^{1 / 3} D$ for solution $\mathrm{A}(\mathbf{a})$ and solution B (b) at ambient temperature $\left(T_{0}=26^{\circ} \mathrm{C}\right)$ well describing the non-linear effect of the droplet size on the normalized fluorescence ratio $R_{12} / R_{120}$. In the case of solution A (Fig. 11a), for the highest $C^{1 / 3} D$ values, significant fluorescence re-absorption can be pointed out. For example, when $C^{1 / 3} D=7$, the decrease of the fluorescence ratio compared to those in cell is about $2.5 \%$

Following the above statements, a corrected model for a fluorescing droplet can be proposed:

$I_{f i}=K_{\mathrm{opt}, i} K_{\mathrm{spec}, i} V_{c} I_{o} C g_{i}\left(C^{1 / 3} D\right) f_{i}(T)$

where $g_{i}$ is an empirical function that takes into account the combined non-linear dependence of the droplet size and dye concentration. The model of Eq. (7) assumes that the effect of the functions $f_{i}$ (temperature dependence) and $g_{i}$ (dependence on the parameter $C^{1 / 3} D$ ) are independent. In order to validate this assumption, the evolution of the fluorescence ratio was recorded as a function of the droplet size with a higher injection temperature $\left(T_{2}=51^{\circ} \mathrm{C}\right)$. This experiment was carried out only for solution $\mathrm{B}\left(C=10^{-6}\right.$ $\mathrm{mol} / \mathrm{l}$ ) because droplet cooling is more limited for n-decane which is less volatile than water. Thus, the droplet temperature will be assumed equal to the liquid injection temperature.

From the normalized fluorescence ratio obtained at $T_{2}=51^{\circ} \mathrm{C}$ and $T_{1}=26^{\circ} \mathrm{C}$ :

$R_{12}\left(T_{1}\right)=\frac{K_{\mathrm{opt}, 1} K_{\mathrm{spec}, 1} f_{1}\left(T_{1}\right)}{K_{\mathrm{opt}, 2} K_{\mathrm{spec}, 2} f_{2}\left(T_{1}\right)} \cdot \frac{g_{1}}{g_{2}}$

$R_{12}\left(T_{2}\right)=\frac{K_{\mathrm{opt}, 1} K_{\mathrm{spec}, 1} f_{1}\left(T_{2}\right)}{K_{\mathrm{opt}, 2} K_{\mathrm{spec}, 2} f_{2}\left(T_{2}\right)} \cdot \frac{g_{1}}{g_{2}}$

and using the reference in cell:

$R_{120}\left(T_{0}\right)=\frac{K_{\mathrm{opt}, 1} K_{\mathrm{spec}, 1} f_{1}\left(T_{0}\right)}{K_{\mathrm{opt}, 2} K_{\mathrm{spec}, 2} f_{2}\left(T_{0}\right)} \frac{g_{10}}{g_{20}}$

it is possible to derive $g_{1} / g_{2}$ by following :

$\frac{g_{1}}{g_{2}}=\frac{R_{12}\left(T_{i}\right)}{R_{120}} \frac{f_{2}\left(T_{i}\right)}{f_{1}\left(T_{i}\right)} \frac{f_{1}\left(T_{0}\right)}{f_{2}\left(T_{0}\right)}\left(T_{i}=T_{1}\right.$ or $\left.T_{2}\right)$

where $g_{10}=g_{20}=1$ in the reference cell (no size effect). Evolution of $g_{1} / g_{2}$ versus $D$ is displayed in Fig. 12 for the two temperatures. The reasonable superimposition of curves determined for the two injection temperatures tends to demonstrate that the functions $f_{i}$ and $g_{i}$ are independents and justify Eq. (7).

However, if the LIF technique thermometry is used directly in a spray for droplet temperature measurements with only two spectral bands $i$ and $j$, it is necessary to know the ratio between $g_{i}$ and $g_{j}$. To solve it, a third spectral band is used. A whole description of the modified LIF technique, and some temperature measurements in a spray, could be found in (Labergue et al. 2008). 


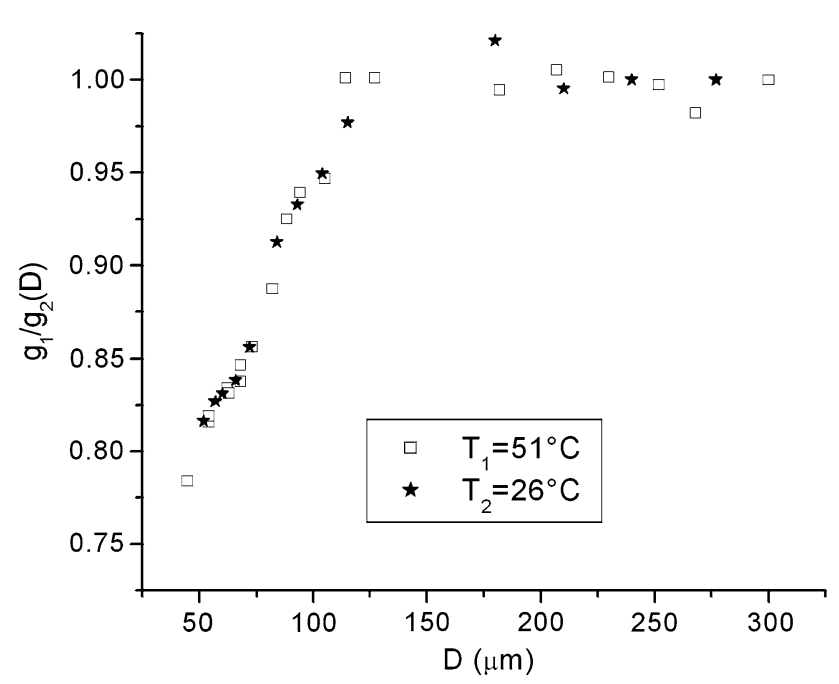

Fig. 12 Evolution of $g_{1} / g_{2}$ derived for two injection temperatures as a function of the droplet diameter (solution $\mathrm{B}$ )

\section{Assumptions about the non-linear dependence of the droplet size}

Modification of the spectral distribution of the fluorescence observed in a spray is directly related to the summation of the individual contribution of each droplet crossing the probe volume. Even if the phenomenon still remains unexplained, some assumptions about the origin of the droplet size effect on the fluorescence spectral distribution can be discussed. Modification of the fluorescence emission spectrum due to photodecomposition of the tracer molecules was excluded, even if the lensing effect of the air-droplet interface is considered: the transit time of the droplet in the laser excitation volume, a few microseconds, is certainly too low to induced any photodecomposition phenomena.

Among the potential causes of the droplet size effect, one could be related to the photophysics of the fluorescent molecules within the droplets. It has been previously shown that chemical reactions can occur at the interface, the reaction rate being dependant on the interface curvature (i.e. droplet diameter). For instance, Habuchi et al. (2000) observed quenching of rhodamine $6 \mathrm{G}$ monomer excited state by the dimer. Although the experimental conditions are drastically different compared to these of the present work (smaller droplets made of ethylene glycol/water mixtures and fixed within a polymer matrix in Habuchi et al. (2000), the dimer formation is strongly dependent on droplet-size and dye concentration. Furthermore, dimer formation is able to modify both fluorescence lifetime and spectral distribution.

\section{Conclusions}

A new phenomenon, related to the use of two-color laserinduced fluorescence thermometry was highlighted.
Fluorescence signal measurements on designated spectral bands and spectral investigations have demonstrated that the droplet size can influence significantly the spectral distribution of fluorescence, especially when the droplet diameter is small. Furthermore, the increase in the dye concentration tends to limit this effect. A new model was proposed for the fluorescence emitted by the droplet. In this model, an empirical function of the parameter $C^{1 / 3} D$ is introduced. This function depends on both fluorescent dye and solvent of interest. An interesting side consequence of this model concerns the case of evaporating or combusting droplets (Castanet et al. 2003 and Castanet et al. 2005). In these cases, the droplets diameter decreases continuously due to the evaporation of the volatile fuel. However, during the evaporation, the fluorescent tracer remains in the liquid phase, as its concentration increases. Consequently, the quantity $C^{1 / 3} D$ remains constant during the evaporation process. Then, the measured temperature evolution is not affected by the influence of the variation of the droplet size during evaporation.

Currently, main studies are focused on the interpretation of this new phenomenon, by performing more accurate spectral studies or measurements of fluorescence lifetime.

\section{References}

Bazile R, Stepowski D (1994) Measurements of the vaporization dynamics in the development zone of a burning spray by planar laser-induced fluorescence and Raman scattering. Exp Fluids 16:171-180

Bruhat G (1992) Fluorescence and phosporescence. In: Cours de Physique Générale, Optique, Kastler, Paris, Milan, Barcelonne, Bonn

Castanet G, Lavieille P, Lebouche M, Lemoine F (2003) Measurement of the temperature distribution within monodisperse combusting droplets in linear streams using two-color laserinduced fluorescence. Exp Fluids 35:563-571

Castanet G, Lebouché M, Lemoine F (2005) Heat and mass transfer of combusting monodisperse droplets in linear stream. Int J Heat Mass Transfer 48:3261-3275

Castanet G, Lienard T, Lemoine F (2009) Dynamics and temperature of droplets impacting onto a heated wall. Int $\mathrm{J}$ Heat Mass Transfer 52:670-679

Depredurand V, Miron P, Labergue A, Wolff M, Lemoine F (2008) A temperature-sensitive tracer suitable for two-color laser-induced fluorescence thermometry applied to evaporating fuel droplets. Meas Sci Technol 19:1-12

Gaskey S, Vacus P, David R, Villermaux J, Andrej C (1990) A method for the study of turbulent mixing using fluorescence spectroscopy. Exp Fluids 9:137-147

Gossage HE, Melton LA (1987) Fluorescence thermometers using intermolecular exciplex. Appl Opt 26:2256-2259

Habuchi S, Kim HB, Kitamura N (2000) Chemical size-effect on fluorescence lifetime of rhodamine $6 \mathrm{G}$ in ethylene glycol/water microdroplets in polydimethylsiloxane matrix. J Photochem Photobio 133:189-196

Kim HJ, Kihm KD, Allen JS (2003) Examination of a ratiometric laser induced fluorescence thermometry for microscale spatial measurement resolution. Int J Heat Mass Transfer 46:3967-3974 
Koenig G, Anders K, Frohn A (1986) A new light-scattering technique to measure the diameter of periodically generated moving droplets. J Aerosol Sci 17:157-167

Labergue A, Delconte A, F Lemoine (2008) Three-color laser induced fluorescence applied to temperature measurements in sprays. 14th Symposicum on applications of laser techniques to fluid mechanics, paper 10.2_4, Lisbon, Portugal

Lavieille P, Lemoine F, Lebouché M, Lavergne G (2001) Evaporating and combusting droplet temperature measurements using twocolor laser-induced fluorescence. Exp Fluids 31:45-55

Lefebvre AH (1989) Atomization and spray. Hemisphere Publishing Corporation, New York

Lemoine F, Antoine Y, Wolff M (1999) Simultaneous temperature and $2 \mathrm{D}$ velocity measurements in a turbulent heated jet using combined laser-induced fluorescence and LDA. Exp Fluids 26:315-323

Maqua C, Castanet G, Grisch F, Lemoine F, Kristiadi T, Sazhin SS (2008) Monodisperse droplet heating and evaporation: experimental study and modeling. Int J Heat Mass Transfer 51:39323945
Murray MA, Melton LA (1985) Fluorescence methods for determination of temperature in fuel spray. Appl Opt 24:2783-2787

Sakakibara J, Adrian RJ (1999) Whole field measurement of temperature in water using two-color laser induced fluorescence. Exp Fluids 26:7-15

Sakakibara J, Hishida K, Maeda M (1997) Vortex structure and heat transfer in the stagnation region of an impinging plane jet. Int $\mathrm{J}$ Heat Mass Transfer 40:3163-3176

Sazhin SS (2006) Advanced models for fuel droplets heating and evaporation. Pro Energy Comb Sci 32:162-214

Van Beeck J, Grosges T, De Giorgi MG (2003) Global rainbow thermometry assessed by airy and Lorenz-Mie theories and compared with phase doppler anemometry. Appl Opt 42:40164022

Vetrano MR, Gauthier S, Beek J, Boulet P, Buchlin JM (2006) Characterization of a non-thermal water spray by global rainbow thermometry. Exp Fluids 40:15-22 